\title{
A Way to Deal With Model-Plant Mismatch for a Reliable Diagnosis in Transient Operation
}

e-mail: s.borguet@ulg.ac.be

\author{
P. Dewallef \\ e-mail: p.dewallef@gmail.com \\ 0. Léonard \\ e-mail: 0.leonard@ulg.ac.be \\ Turbomachinery Group, \\ University of Liège, \\ Chemin des Chevreuils 1 , \\ 4000 Liège, Belgium
}

Least-squares health parameter identification techniques, such as the Kalman filter, have been extensively used to solve diagnosis problems. Indeed, such methods give a good estimate provided that the discrepancies between the model prediction and the measurements are zero-mean, white, Gaussian random variables. In a turbine engine diagnosis, however, this assumption does not always hold due to the presence of biases in the model. This is especially true for a transient operation. As a result, the estimated parameters tend to diverge from their actual values, which strongly degrades the diagnosis. The purpose of this contribution is to present a Kalman filter diagnosis tool where the model biases are treated as an additional random measurement error. The new methodology is tested on simulated transient data representative of a current turbofan engine configuration. While relatively simple to implement, the newly developed diagnosis tool exhibits a much better accuracy than the original Kalman filter in the presence of model biases. [DOI: 10.1115/1.2833491]

Keywords: engine health monitoring, Kalman filter, model-plant mismatch

\section{Introduction}

The diagnosis tool considered herein is basically a gas path analysis method whose purpose is to assess the deviations of some health parameters on the basis of measurements collected within the gas path of the engine [1]. The health parameters are coefficients affecting the efficiency and the flow capacity of the components (fan, low pressure compressor (lpc), high pressure compressor (hpc), high pressure turbine (hpt), low pressure turbine (lpt), and nozzle), while the measurements are intercomponent temperatures, pressures, as well as rotational speeds. The health assessment leads to a diagnosis of the engine condition, which allows suitable maintenance actions to be undertaken.

The health parameter estimation is achieved by a Kalman filter, which is a minimum mean square error (variance) estimator within a recursive framework [2]. This means that the estimated health parameters minimize the distance (in the least-squares sense) between a model prediction and the observed measurements. Moreover, the recursive structure of the Kalman filter updates the values of the identified health parameters as new data are available, which is a useful property in real-world applications such as on-board performance monitoring [3].

Since the first research efforts of Urban [4], most of the gas path analysis methods were restricted to measurements observed under steady-state operation of the engine, mainly for computational load reasons. For a few years, the ability to extract the engine condition from transient data has been investigated using various techniques such as least-squares estimation [5,6], artificial neural networks [7] for a batch treatment of the data, and Kalman filters [8-11] in a recursive framework.

More specifically, it has been shown in Ref. [10] that the use of measurements representative of transient behavior significantly improves both the diagnosis accuracy and the isolation capability under negative redundancy (i.e., more health parameters than sensors), provided that a faithful dynamic model is available. Indeed, a transient operation allows a much greater number of operating points to be considered, thereby increasing the analytical redundancy.

Manuscript received June 20, 2006; final manuscript received October 29, 2007; published online March 26, 2008. Review conducted by Jeffrey W. Bird.
Although the existence of a perfectly faithful model is generally assumed, this hypothesis is rarely met in practice. In fact, complex phenomena, such as heat transfer, volume dynamics, clearances, and secondary airflow and power off-takes, are poorly modeled or unmodeled in current state-of-the-art aerothermodynamic engine models $[12,13]$. Consequently, the performance predictions generated by the dynamic model are biased with respect to measurements taken on the engine. As reported in Ref. [11], those biases strongly reduce the efficiency of the diagnosis tool.

The present contribution proposes a solution to the model biases by considering them as an additional measurement error. Indeed, from the point of view of an external observer, it is not possible to distinguish a model bias from a sensor error. However, unlike sensor errors, which are basically unpredictable beforehand, the model biases of interest have a more predictable nature that can be studied by comparing the model outputs and the measurements observed on a healthy engine during a learning phase previous to the health parameter assessment.

\section{Description of the Method}

The scope of this section is to provide a short description of our diagnosis tool and to present the methodology we have developed to cope with model-plant mismatch and its integration within the diagnosis algorithm.

Diagnosis Tool. Our diagnosis tool belongs to the family of model-based approaches, meaning that a simulation model of the turbine engine must be available. In the framework of gas path analysis, these are basically nonlinear aerothermodynamic models based on mass, energy, and momentum conservation laws applied to the engine. ${ }^{1}$

As mentioned in the Introduction, the framework in which this contribution takes place is the development of a diagnosis tool processing transient data and reliance on the Kalman filter estimation algorithm. Since the system model is nonlinear, the unscented Kalman filter [14] is used instead of the generic linear Kalman filter. A few adjustments and assumptions govern the applicability

${ }^{1}$ Linearized models are often used to lower the computational burden. 
of the unscented Kalman filter. The first one consists in formulating the simulation model of the engine in terms of a state-space model, namely,

$$
\begin{gathered}
\mathbf{x}_{k}=\mathcal{F}\left(\mathbf{u}_{k}, \mathbf{v}_{k}, \mathbf{w}_{k}, \mathbf{x}_{k-1}\right)+\boldsymbol{\nu}_{k} \\
\mathbf{y}_{k}=\mathcal{G}\left(\mathbf{u}_{k}, \mathbf{v}_{k}, \mathbf{w}_{k}, \mathbf{x}_{k}\right)+\boldsymbol{\epsilon}_{k}
\end{gathered}
$$

where $k$ is a discrete time index, $\mathbf{u}_{k}$ are the command parameters (e.g., fuel flow), $\mathbf{v}_{k}$ are measurable exogenous inputs (e.g., Mach number and altitude), $\mathbf{w}_{k}$ are the aforementioned health parameters, and $\mathbf{x}_{k}$ are the state variables. The state variables are intended to handle the transient effects taking place in the gas path of the engine. Generally speaking, these transient effects belong to three categories, namely, the heat transfers between the gas path and the components of the engine, the shaft inertia, and the fluid transport delays.

Equation (2) is called the measurement equation and joins the deterministic simulation model $\mathcal{G}(\cdot)$ and a random variable $\boldsymbol{\epsilon}_{k}$ intended to represent the stochastic influence of the measurement uncertainties. Similarly, Eq. (1) is named the state prediction equation and links the deterministic integration routine $\mathcal{F}(\cdot)$ of the simulation model and a random variable $\boldsymbol{\nu}_{k}$, which represents an error term. In addition to these two equations, a third one, named the health parameter transition equation, is often included which states that the health parameters may vary in time by following a first-order Markov process (see Ref. [3] for further details).

The second assumption is that both $\boldsymbol{\nu}_{k}$ and $\boldsymbol{\epsilon}_{k}$ are zero-mean, white, and Gaussian random variables ${ }^{2}$ which is denoted by

$$
\boldsymbol{\epsilon}_{k}=\mathcal{N}\left(\mathbf{0}, \mathbf{R}_{\mathbf{y}}\right) \text { and } \boldsymbol{\nu}_{k}=\mathcal{N}\left(\mathbf{0}, \mathbf{R}_{\mathbf{x}}\right)
$$

Since the state variables are unknown (not all of them are measurable), they must be estimated together with the health parameters from the same sequence of measurements $\mathbf{y}_{k}$. This problem, known as the dual estimation problem, is solved herein by a socalled dual estimation Kalman filter (DEKF). This dual Kalman filter relies on two unscented Kalman filters running concurrently, one for the health parameters and the other for the state variables. The interested reader may consult Ref. [15] for a detailed description of the algorithm. Basically, once the former filter has updated the health parameters, the current value is used by the latter to update the corresponding state variables.

Provided that a prior value for the health parameters and the state variables is available, the basic step consists of observing the discrepancies between the model outputs, denoted by $\hat{\mathbf{y}}_{k}$, and the observed measurements $\mathbf{y}_{k}$. These discrepancies, also called residuals and denoted by $\mathbf{r}_{k}$, are processed by the DEKF, which recursively updates the health parameters and the state variables so that the average value of $\mathbf{r}_{k}$ is driven to zero. In other words, the identified health parameters provide a means of observing the actual health condition of the engine. Figure 1 summarizes this recursive, closed-loop process. The engine performance model, actually embedded in the DEKF, has been represented outside of it in order to underline its important role in the estimation process.

Dealing With Model Biases. The physical meaning of the identified health parameters is only valid provided that the model is faithful. Otherwise, as reported by Volponi in Ref. [11], the health parameters become "tuners," which are adjusted by the identification process to fit the behavior of the real engine, losing sense for diagnosis reports. Indeed, as formulated in the preceding section, the Kalman filter assumes that the discrepancies between the model and the measurements are zero on average.

Unfortunately, model biases do not have a pure stochastic nature, but should rather be seen as systematic errors whose mean values are different from zero. The assumption on the noise characteristics is therefore violated, which perturbs the health param-

\footnotetext{
${ }^{2}$ In addition, $\boldsymbol{\nu}_{k}$ and $\boldsymbol{\epsilon}_{k}$ are assumed uncorrelated.
}

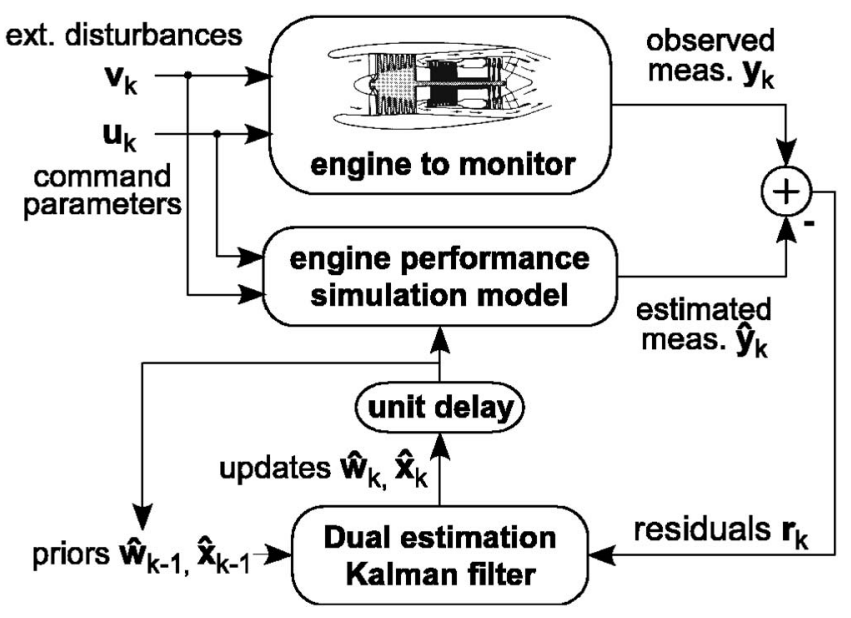

Fig. 1 Health parameter and state variable update mechanism using a DEKF

eter identification. In practice, it has been observed that model biases as small as the measurement noise standard deviation can dangerously reduce the reliability of the diagnosis. Therefore, the biases have to be modeled in some way so that the residuals will reflect only the degradation of the engine. In reality, the modelplant mismatch depicts the approximations made in both the state transition equation (Eq. (1)) and the measurement equation (Eq. (2)). A possible mathematical translation of this fact is to consider that neither $\boldsymbol{\epsilon}_{k}$ nor $\boldsymbol{\nu}_{k}$ are zero-mean, Gaussian random variables.

In this contribution, it is proposed to gather all the effects caused by modeling errors in the single measurement equation (Eq. (2)). The measurement noise $\boldsymbol{\epsilon}_{k}$ is now seen as a hybrid bias-noise term gathering the measurement noise and the modelplant mismatch. Mathematically speaking, $\boldsymbol{\epsilon}_{k}$ is considered as a Gaussian random variable of variable properties (i.e., mean and covariance). Two reasons favored this choice: First, the Kalman filter deals with this type of random variables. Second, a Gaussian random variable is totally defined by its mean and its covariance, which is simple to handle in practice,

$$
\boldsymbol{\epsilon}_{k}=\mathcal{N}\left(\mathbf{b}_{k}, \mathbf{R}_{\mathbf{b}, \mathbf{k}}\right)
$$

Provided that $\mathbf{b}_{k}$ and $\mathbf{R}_{\mathbf{b}, \mathbf{k}}$ are known, the mechanism of the unscented Kalman filter can be applied by making the following substitutions:

$$
\begin{gathered}
\mathbf{r}_{k}=\mathbf{y}_{k}-\hat{\mathbf{y}}_{k} \rightarrow \mathbf{r}_{k}=\mathbf{y}_{k}-\hat{\mathbf{y}}_{k}-\mathbf{b}_{k} \\
\mathbf{R}_{\mathbf{y}} \rightarrow \mathbf{R}_{\mathbf{b}, \mathbf{k}}
\end{gathered}
$$

Determination of $\mathbf{b}_{\boldsymbol{k}}$ and $\boldsymbol{R}_{\mathbf{b}, \mathbf{k}}$. As already mentioned above, the model biases are not, strictly speaking, random variables. Consequently, they can be studied beforehand, for example, during the acceptance tests that every engine undergoes before it is brought into service. The purpose of this offline learning is to build a model that predicts $\mathbf{b}_{k}$ and $\mathbf{R}_{\mathbf{b}, \mathbf{k}}$ as precisely as possible. This approach is very close to the eStorm philosophy [11], with the difference that in our study both the mean bias and its uncertainty are modeled.

During this learning phase, model outputs are compared to the observed measurements without estimating the health parameters, which are assumed to be at their healthy nominal values. As the engine transient model is not perfect, the observed residuals $\mathbf{r}_{k}$ correspond to the model biases. The next step of the learning phase consists of characterizing the mean $\mathbf{b}_{k}$ and the covariance matrix $\mathbf{R}_{\mathbf{b}, \mathbf{k}}$ of the observed biases. 
Modeling the mean bias $\mathbf{b}_{k}$ is typically a function approximation problem. In the following, some general considerations about this problem are recalled. Numerous textbooks address this problem in a more exhaustive way (see, for instance, Ref. [16]).

The cloud of data of the learning set has to be fitted with a function $\mathcal{H}$, which may depend on the controls, the ambient variables, the state variables, and, possibly, past values of them and which also contains fitting parameters $\mathbf{p}$, as stated in

$$
\mathbf{b}_{k}=\mathcal{H}\left(\mathbf{u}_{k}, \mathbf{v}_{k}, \mathbf{x}_{k}, \ldots, \mathbf{p}\right)
$$

The first step is to decide on which input variables the function $\mathcal{H}$ depends, with the aim of reducing the dimensionality of the input space by performing a so-called feature extraction. A feature can be seen as an intelligent transformation of the original input variables. Then, the structure (e.g., polynomial fit or neural networks) and the complexity (e.g., order of the polynomial or number of hidden nodes) have to be selected. As a result, the number of fitting parameters $\mathbf{p}$ is obtained. For example, in the case of a scalar, second-order polynomial fit, the fitting parameters $\mathbf{p}$ are the three coefficients of the parabola.

The choice of the flexibility of the fitting function must be done in a very careful way. Indeed, the goal is to obtain the best representation of the underlying properties of the data in the learning set and hence to obtain the best generalization performance. In other words, over-fitting of the data must be avoided. So, the number of samples in the learning set should be large enough with respect to the flexibility of the approximation function. On the other hand, a general result of learning theory states that for a given number of fitting parameters, the larger the database, the more meaningful the values of these parameters from a statistical point of view (see Ref. [16]).

The fitting parameters $\mathbf{p}$ are generally computed through the minimization of an error function (e.g., sum of squares error). The determination of the covariance matrix $\mathbf{R}_{\mathbf{b}, \mathbf{k}}$ is to be explained later.

Considering the present application, a rather basic, but physically meaningful, model is chosen. First, fixed ambient conditions (e.g., sea-level static) are selected when collecting the learning set. Then, three assumptions are made in order to simplify the determination of $\mathbf{b}_{k}$ and $\mathbf{R}_{\mathbf{b}, \mathbf{k}}$.

1. The engine steady-state model is highly accurate. To this end, model-matching techniques such as those described in Refs. [17] or [18] can be applied.

2. The engine undergoes moderate transient maneuvers. This constraint can be expressed in terms of an upper limit on the engine acceleration. This bound is application dependent and was set here for the sake of simplicity to a value of $\pm 200 \mathrm{rpm} / \mathrm{s}$ on the fan rotor acceletation.

3. During the learning phase, the engine is in healthy nominal condition, hence the values of the health parameters are known and set to their nominal values.

The problem of modeling the mean $\mathbf{b}_{k}$ is first investigated. We can reasonably suppose that the more rapid and complex the transient is, the greater the model-plant mismatch is. Therefore, it is desirable to link the mean bias $\mathbf{b}_{k}$ to a scalar quantity, which is representative of the "intensity" of the transient and which is easy to compute. To this end, the following transient index (TI) is defined:

$$
\mathrm{TI}_{k}=\frac{1}{n_{x}} \sum_{i=1}^{n_{x}} \frac{\dot{\hat{\mathbf{x}}}_{k}(i)}{\mathbf{x}^{\mathrm{ref}}(i)}
$$

where $n_{x}$ is the number of state variables of the on-board model, $\dot{\hat{\mathbf{x}}}_{k}(i)$ is the derivative of state variable $i$ at time index $k$, and $\mathbf{x}^{\mathrm{ref}}(i)$

${ }^{3}$ Note that another indicator of engine acceleration could be chosen, e.g., core acceleration.

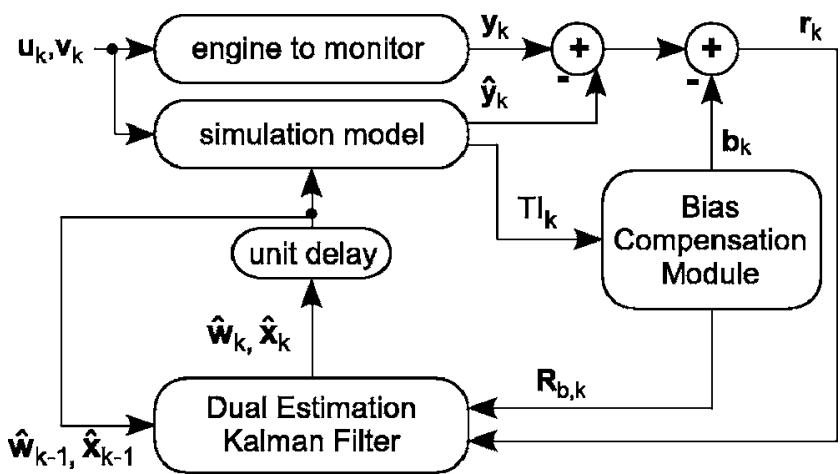

Fig. 2 Integration of the $\mathrm{BCM}$

is the reference value of state variable $i$ (e.g., at take-off rating). The unit of TI is $\mathrm{s}^{-1}$. The normalization by a reference state value is required given the different orders of magnitude of the state variables. The TI is zero in a steady-state operation, positive when the engine is accelerating, and negative otherwise.

The TI is computed based on state derivatives provided by the engine model rather than on actual measurements. Two reasons dictate this choice: First, the engine model produces noise-free signals and outputs directly the state derivatives; second, not all state variables are normally measurable on the real engine (e.g., the metal temperatures involved in the heat transfers) but are available in the engine model.

So, modeling the mean value of the bias amounts to determining the mapping $\mathbf{b}_{k}=f\left(\mathrm{TI}_{k}\right)$ from the database of biases. This problem is solved through a least-squares polynomial fit. The selection of the polynomial order and a possible partitioning of the TI axis is a question of engineering judgment. Additional indications are provided in the application detailed later in the paper.

The model of the mean value of the bias available, the covariance matrix of the bias, $\mathbf{R}_{\mathbf{b}, \mathbf{k}}$, can now be computed. The procedure is given in Algorithm 1. Depending on the partitioning adopted for the mean bias, one covariance matrix is computed per TI segment. As a first step, the gap between each data point and the mean bias is computed for all data points belonging to a particular segment (lines $2-5$, note that each vector $\mathbf{e}_{i}$ is a $n y \times 1$ vector). Finally, each element of the symmetric covariance matrix is obtained using the well-known definition of the covariance of random variables (line 6).

$$
1 .
$$

Algorithm 1. Covariance matrix computation.

2. 3. 4. 5.

6.

$$
\begin{aligned}
& \text { Set } N=0 \\
& \text { for all } k \text { such that } \mathrm{TI}^{\min }<\mathrm{TI}_{k} \leqslant \mathrm{TI}^{\max } \text { do } \\
& \qquad N=N+1 \\
& \quad \mathbf{e}_{N}=\hat{\mathbf{r}}_{k}-\mathbf{b}\left(\mathrm{TI}_{k}\right) \\
& \text { end for } \\
& \mathbf{R}_{\mathbf{b}}=\frac{1}{N-1} \sum_{i=1}^{N}\left\{\mathbf{e}_{i} \mathbf{e}_{i}^{T}\right\}
\end{aligned}
$$

The covariance matrix takes into account both the measurement noise (sensor inaccuracies) and the possible variability of the mean bias. Also, it should not be surprising that some off-diagonal terms of that matrix are nonzero. This simply indicates that the sensor biases are interdependent as the modeling errors introduce some relationships between the measurements: for instance, the bias on the exhaust gas temperature (EGT) sensor measurement is linked to that of the low pressure spool speed since temperature recovery factor for thermocouples will vary with mass flow.

This concludes the offline modeling of the bias model, which can now be integrated within the diagnosis algorithm in order to make it more robust to model-plant mismatches.

Modification of the Diagnosis Algorithm. The block diagram of the modified diagnosis algorithm is shown in Fig. 2. A short 


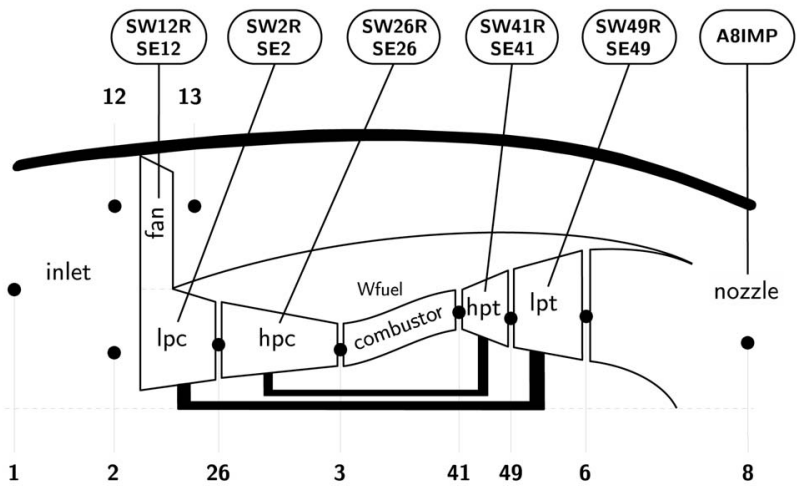

Fig. 3 Turbofan layout with station numbering and health parameter location

description of the procedure is given in this section. Similar to the original procedure detailed in Fig. 1, the previous estimates of the state variables $\hat{\mathbf{x}}_{k-1}$ and health parameters $\hat{\mathbf{w}}_{k-1}$ are used together with the current inputs $\mathbf{u}_{k}$ and $\mathbf{v}_{k}$ by the engine performance model to generate an estimation of the measurements. Additionally, the $\mathrm{TI}_{k}$ is computed using relation (8). From this TI, the mean bias $\mathbf{b}_{k}$ and its covariance matrix $\mathbf{R}_{\mathbf{b}, \mathbf{k}}$ are obtained according to the bias model previously set up. The bias $\mathbf{b}_{k}$ is taken into account in the residual $\mathbf{r}_{k}$, which is fed into the original DEKF, with the only difference that the measurement noise covariance matrix $\mathbf{R}_{\mathbf{y}}$ is replaced by $\mathbf{R}_{\mathbf{b}, \mathbf{k}}$. Loosely speaking, the mean bias $\mathbf{b}_{k}$ and its covariance matrix $\mathbf{R}_{\mathbf{b , k}, \mathbf{k}}$ are now extra inputs to the original DEKF. The integration of the bias compensation module (BCM) within the diagnosis algorithm has a very limited impact on the computational load. Indeed, only a polynomial evaluation is used to return $\mathbf{b}_{k}$ and $\mathbf{R}_{\mathbf{b}, \mathbf{k}}$.

\section{Application of the Method}

Engine Configuration. The application used as a test case is a high bypass ratio mixed-flow turbofan. The engine layout is described in Fig. 3 where the health parameter location and the station numbering are also indicated. One command variable, which is the fuel flow rate fed in the combustor, is considered in the following.

The engine performance model has been developed and validated as part of the OBIDICOTE ${ }^{4}$ project. A detailed description of the model can be found in Ref. [19].

As real data were not available, we worked with simulated data only. To introduce modeling errors, we considered two different configurations of the OBIDICOTE model with regard to transient operation. Our hypothesis concerning the perfect fidelity of the steady-state model to the data used is hence implicitly satisfied.

The sensor configuration adopted in the test cases is representative of typical instrumentation available on modern turbofan engines and is detailed in Table 1. The stated uncertainties have been selected according to the OBIDICOTE documentation and account for the magnitude of random errors only.

"Real" Engine and On-Board Model. The OBIDICOTE model plays the role of the "real" engine. The shaft dynamics and the heat transfers in the hpc, burner, and hpt are thus supposed to be perfectly modeled. The seven state variables involved in this first model are listed in Table 2.

Gaussian noise, whose magnitude is specified in Table 1, is added to the clean measurements generated by the real engine model to make them closer to typical test data. The sampling rate

${ }^{4} \mathrm{~A}$ Brite/Euram project for on-board identification, diagnosis, and control of turbofan engine.
Table 1 Sensor configuration and assumed uncertainty

\begin{tabular}{cc}
\hline \hline Label & Uncertainty \\
\hline$T_{13}$ & $\pm 2 \mathrm{~K}$ \\
$P_{13}$ & $\pm 100 \mathrm{~Pa}$ \\
$T_{3}$ & $\pm 2 \mathrm{~K}$ \\
$P_{3}$ & $\pm 5000 \mathrm{~Pa}$ \\
$N_{\mathrm{lp}}$ & $\pm 6 \mathrm{rpm}$ \\
$N_{\mathrm{hp}}$ & $\pm 12 \mathrm{rpm}$ \\
$T_{6}$ & $\pm 2 \mathrm{~K}$ \\
\hline \hline
\end{tabular}

is set to $50 \mathrm{~Hz}$, which is a typical value to capture the transient effects described above.

A second model is embedded in the diagnosis algorithm and plays the role of the imperfect on-board model. It suffers from model-plant mismatch since the heat transfer processes are poorly modeled with respect to the real engine. As shown in Table 3, only three state variables are involved in this second model. They are related to the spool inertia and to a bulk heat transfer in the hpt.

Heat transfer processes are the slowest dynamics in a turbine engine and they quite strongly influence the transient response of the engine (see Ref. [13]). Considering the present application, the heat transfer is placed on the hpt since it is the hottest part of the engine; therefore, the thermal effects are expected to be more important than in other components. Moreover, the observability of the hpt thermal state is satisfactory with the selected sensor suite (see Ref. [10]).

Modeling the Bias. The methodology for bias modeling described in a previous section is applied to the turbofan layout. The task of bias modeling is obviously application dependent. Reported here are the main issues of the process for the real engine and on-board model setup.

The first step is to build a database of residuals for the healthy engine, which will serve as a learning set for determining the bias model. Figure 4 depicts the fuel flow trajectory input to the real engine and the on-board model. In this contribution, the engine model is run in open loop; therefore, the set point is specified in terms of fuel flow rather than fan speed or engine pressure ratio. For reference, the lowest fuel flow value (slightly less than $0.2 \mathrm{~kg} / \mathrm{s}$ ) corresponds to the ground idle rating, and the greatest one (slightly higher than $1.2 \mathrm{~kg} / \mathrm{s}$ ) gives the take-off power rating. The sequence is $1900 \mathrm{~s}$ long so that the database contains 95,000 samples (per sensor). Other scenarios could be added to the database.

Table 2 State variables for the real engine

\begin{tabular}{cl}
\hline \hline Label & \multicolumn{1}{c}{ Description } \\
\hline$N_{\mathrm{lp}}$ & Low pressure spool rotational speed \\
$N_{\mathrm{hp}}$ & High pressure spool rotational speed \\
$T_{m 3 b}$ & hpc blade temperature \\
$T_{m 3 c}$ & hpc casing temperature \\
$T_{m 4 b}$ & Combustion chamber casing temperature \\
$T_{m 42 b}$ & hpt blade temperature \\
$T_{m 42 c}$ & hpt casing temperature \\
\hline \hline
\end{tabular}

Table 3 State variables for the on-board model

\begin{tabular}{cl}
\hline \hline Label & \multicolumn{1}{c}{ Description } \\
\hline$N_{\mathrm{lp}}$ & Low pressure spool rotational speed \\
$N_{\mathrm{hp}}$ & High pressure spool rotational speed \\
$T_{m 42}$ & hpt metal temperature \\
\hline
\end{tabular}




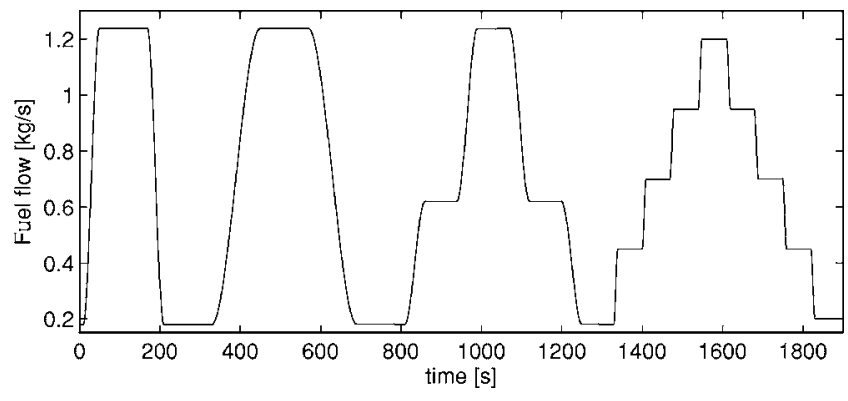

Fig. 4 Fuel flow profile for learning set generation

The mismatch between the real engine and the on-board model appears in Fig. 5, where the normalized root mean square error (NRMSE) is plotted for each sensor. The NRMSE for the $i$ th sensor is defined according to

$$
\operatorname{NRMSE}(i)=\sqrt{\frac{1}{n-1} \sum_{k=1}^{n} \frac{\left(\mathbf{y}_{k}(i)-\hat{\mathbf{y}}_{k}(i)\right)^{2}}{\mathbf{R}_{\mathbf{y}}(i, i)}}
$$

As can be seen in Fig. 5, the NRMSE should be equal to 1 if the on-board model were perfect (white bars). Indeed, in that case, the only source of variation in the response of the real engine and the on-board model is the sensor noise. For the incomplete on-board model considered in this application (gray bars), it can be seen that the prediction for $T_{13}$ is quite faithful; modeling errors, however, have a significant effect on the other measurements and particularly on $T_{3}$. Note that the $T_{3}$ prediction error could be reduced by introducing heat transfer on the hpc.

Now, the job consists in defining a model for the residuals from the real and BCM-off data. Plotted in Fig. 6 is the cloud of $T_{3}$ residuals (black circles) with respect to TI for the operation of the engine under the inputs of Fig. 4.

After examining the residual cloud of each measurement, it was decided to split the TI axis into three distinct regions and to apply a quadratic least-squares fit on the data to determine the mean bias

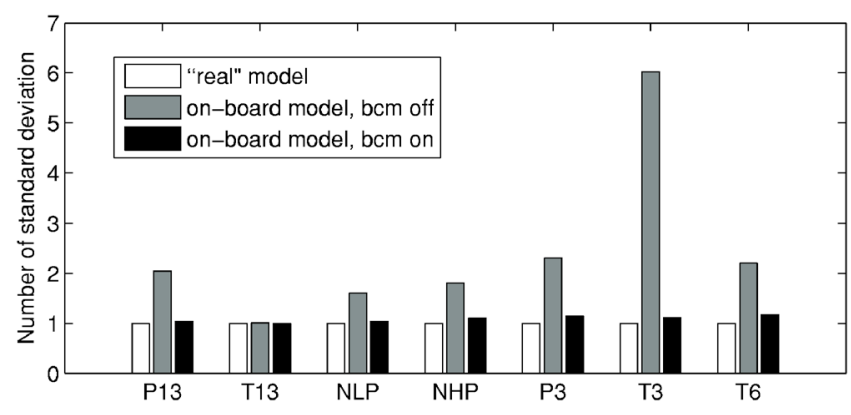

Fig. 5 Model-plant mismatch

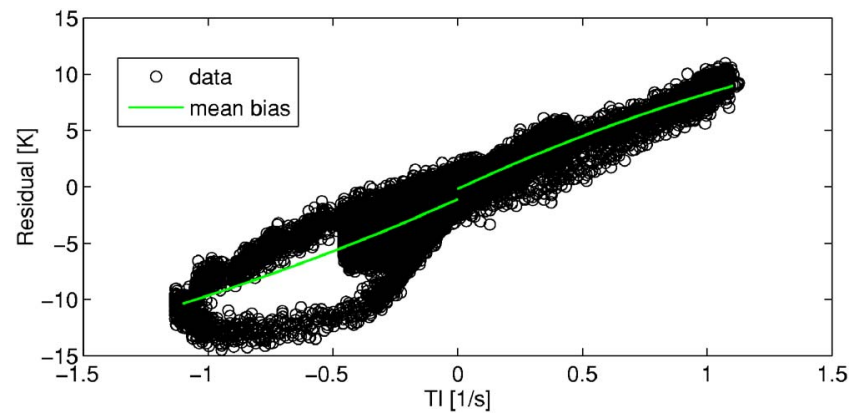

Fig. 6 Mean bias extraction for $T_{3}$
Table 4 Summary of the bias model

\begin{tabular}{lll}
\hline \hline \multicolumn{1}{c}{ Region } & \multicolumn{1}{c}{ Mean bias } & \multicolumn{1}{c}{ Covariance matrix } \\
\hline $\mathrm{TI}_{k}>\mathrm{TI}^{\star}$ & $\mathbf{b}_{k}=\mathbf{p} 1 \mathrm{TI}_{k}^{2}+\mathbf{p 2 T I}+\mathbf{p 3}$ & $\mathbf{R}_{\mathbf{b}, \mathbf{k}}$ per Algorithm 1 \\
$\mathrm{TI}_{\mathbf{k}}<-\mathrm{TI}^{\star}$ & $\mathbf{b}_{k}=\mathbf{p} 4 \mathrm{TI}_{k}^{2}+\mathbf{p 5 T I}+\mathbf{p 6}$ & $\mathbf{R}_{\mathbf{b}, k}$ per Algorithm 1 \\
$\left|\mathrm{TI}_{k}\right|<\mathrm{TI}^{\star}$ & $\mathbf{b}_{k}=\mathbf{0}$ & $\mathbf{R}_{\mathbf{b}, \mathbf{k}}=\mathbf{R}_{\mathbf{y}}$ \\
\hline \hline
\end{tabular}

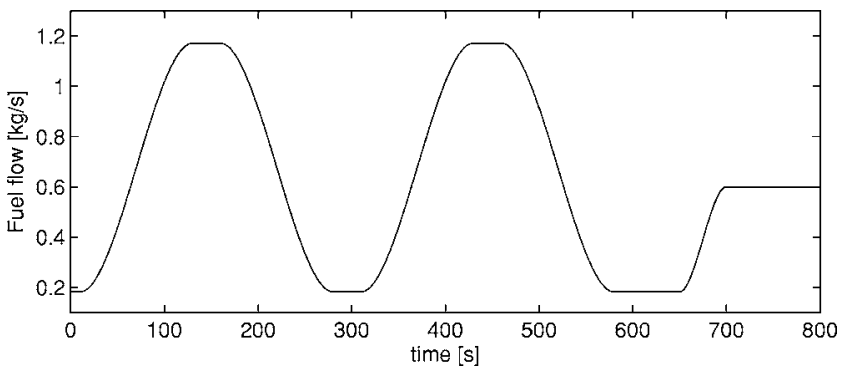

Fig. 7 Fuel flow profile, test bench conditions

(green lines in Fig. 6). The covariance matrices were then computed using Algorithm 1. Higher-order polynomials were rejected since the aim is to extract the global trend in the bias.

The resulting bias model is summarized in Table 4 . TI $^{\star}$ is a threshold value (set to $10^{-3}$ in this application) that makes operating points with small absolute values of TI considered as steadystate ones. For these operating points, the mean bias is set to zero and the covariance matrix $\mathbf{R}_{\mathbf{b}, \mathbf{k}}$ reduces to the original measurement noise covariance matrix $\mathbf{R}_{\mathbf{y}}$ given the assumption of a perfect steady-state model. The vectors p1, p2, p3 and p4, p5, p6 are the coefficients of the quadratic least-squares fit for the $\mathrm{TI}>\mathrm{TI}^{\star}$ and $\mathrm{TI}<-\mathrm{TI}^{\star}$ regions, respectively.

The effect of the BCM on the prediction error can be seen in Fig. 5. The black bars represent the NRMSE for each sensor when the BCM is turned on. Obviously, the simple model defined above enhances the accuracy of the prediction provided by the on-board model as all NRMSEs are closer to unity than when the BCM is disabled (gray bars).

Test-Case A: Diagnosis at Test Bench. To assess the improvements brought by the BCM, the following test case has been developed: It is representative of a maintenance session on a test bench for which sea-level static (SLS), standard day conditions are assumed. The evolution of the fuel flow with respect to time is sketched in Fig. 7. It is an $800 \mathrm{~s}$ sequence made of two successive power sweeps between idle and max-continuous regimes, followed by an acceleration between idle and part-power regimes. ${ }^{5}$

Engine deterioration is simulated from the component fault case proposed in Ref. [20]. It consists of a deviation of nearly all health parameters at $t=0 \mathrm{~s}$ with the following magnitude: $-1.5 \%$ on SW12R, $-1.2 \%$ on SE12, $-1.0 \%$ on SW2R, $-1.0 \%$ on SE2, $-2.3 \%$ on SW26R, $-1.4 \%$ on SE26, $+0.88 \%$ on SW41R, $-1.6 \%$ on SE41, and $-1.3 \%$ on SE49.

The evolution of the health parameters identified with the original DEKF (i.e., with the BCM disabled) is plotted in Fig. 8. The health parameters exhibit an erratic behavior. Clearly, little valuable information about the health condition of the engine can be derived from the graphs. The health parameters are used by the DEKF as tuners to drive the residuals to zero (on average). The health parameters of the hpc and both turbines seem to be particularly sensitive to the model-plant mismatch.

When the BCM is enabled, the identification of the health parameters is depicted in Fig. 9. The improvement with respect to the disabled-BCM case is obvious. The health parameters do not

\footnotetext{
${ }^{5}$ Recall that the engine is open loop, fuel flow piloted.
} 

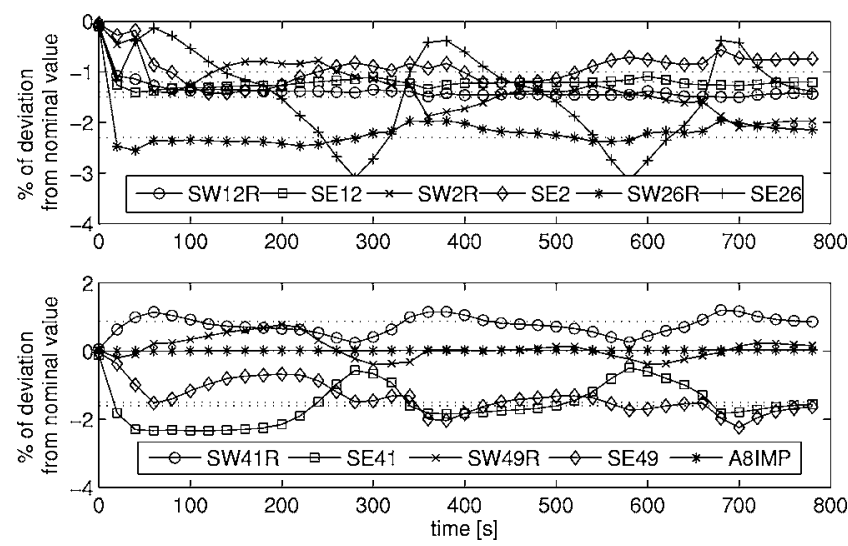

Fig. 8 Diagnosis with $\mathrm{BCM}$ disabled

wander according to the transient but converge to their actual value, and the actual condition of the engine can be stated. About $300 \mathrm{~s}$ is required to converge to the actual engine health as can be noted on the lower graph reporting the health parameters of the hot section. The small variations in SW26R, SE26, and SW41R are due to the remaining modeling error on $T_{3}$ (see Fig. 5).

To underline the originality of our approach that models the mean bias level, and also the uncertainty associated with this bias (through the covariance matrix $\mathbf{R}_{\mathbf{b}, \mathbf{k}}$ ), Fig. 10 depicts the identification of the hpt degradation when using a hybrid BCM setting. The mean bias is computed based on the model presented in Table 4 , but the covariance matrix of the measurement is set to $\mathbf{R}_{\mathbf{b}, \mathbf{k}}$
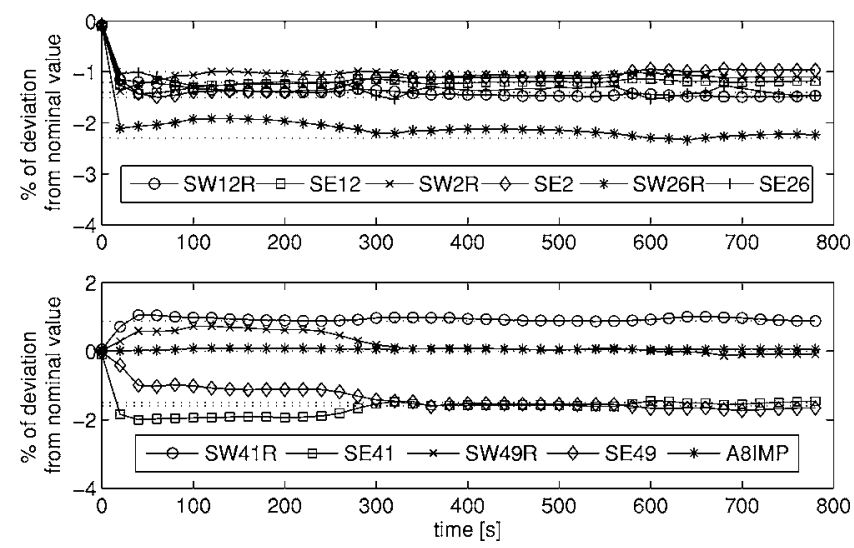

Fig. 9 Diagnosis with $\mathrm{BCM}$ enabled
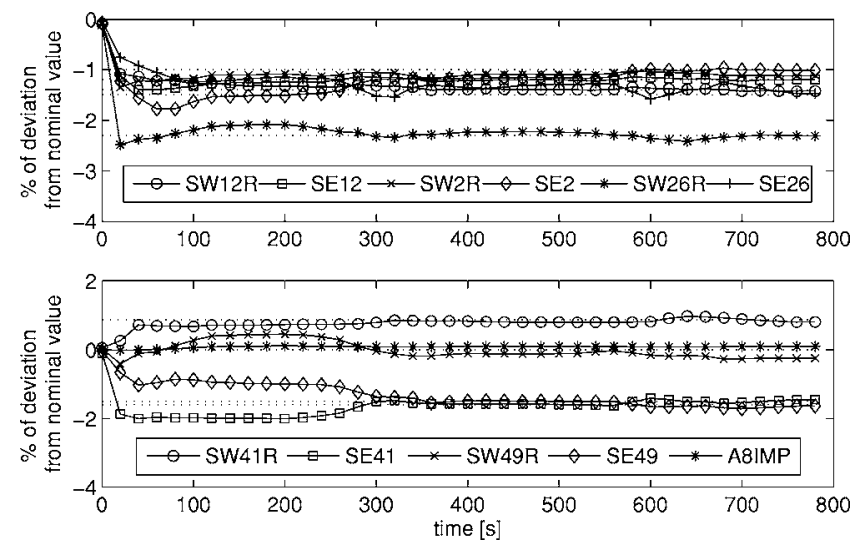

Fig. 10 Diagnosis with hybrid BCM
$=\mathbf{R}_{\mathbf{y}}$, whatever the TI value is. In doing so, no information about the accuracy of the bias $\mathbf{b}_{k}$ is transmitted to the DEKF. This corresponds to the assumption that $\mathbf{b}_{k}$ perfectly matches the model bias.

These results are much better than those presented in Fig. 8 where the BCM was totally disabled. This hints at the fact that the mean bias has indeed the most disruptive effect on the diagnosis algorithm. Yet, some instabilities are still present in Fig. 10, which disappear when the covariance matrix $\mathbf{R}_{\mathbf{b}, \mathbf{k}}$ is used (i.e., in Fig. 9). This can readily be seen by comparing the smoothness of the curves between Figs. 10 and 9.

From this last result, it can be concluded that even if the modeling of the bias is quite simple and not always very accurate, taking the uncertainty in the bias into account can improve the quality of diagnostics. Hence, the role of the covariance matrix $\mathbf{R}_{\mathbf{b}, \mathbf{k}}$ in the Kalman filter algorithm is to deemphasize the influence of the residuals on the health parameter update when a model bias is likely to be expected or when our knowledge about the bias is not very accurate.

Adapting the Bias Compensation Module to Non-Sea-Level Static Conditions. In the previous subsection, the positive effect of the BCM on the diagnosis algorithm has been demonstrated. The diagnosis was performed in the same atmospheric conditions as for the extraction of the bias model (i.e., SLS conditions). Practically, it is indeed difficult to collect biases outside of a pass-off test. Hence, it would be highly valuable to use the predefined $\mathrm{BCM}$ for any other operating conditions.

The extension of the BCM to the whole flight envelope of the engine is nearly immediate by having recourse to the concept of corrected parameters, which relies on similarity laws and firstorder approximation of the gas turbine aerothermodynamic processes. The basic idea behind similarity laws is to define dimensionless groups of parameters that are associated with the flow field in the engine. Those corrected parameters allow a comparison, generally on a Mach number basis, of the performance of the engine operating under different atmospheric conditions. As a reminder, the general expression for the corrected parameter $X$ is given by

$$
X^{c o}=\frac{X}{\theta^{a} \delta^{b}}
$$

where $\quad \theta=T_{2} / T_{\text {ref }}, \quad \delta=P_{2} / P_{\text {ref }}, \quad T_{\text {ref }}=288.15 \mathrm{~K}, \quad$ and $\quad P_{\text {ref }}$ $=101,325 \mathrm{~Pa}$.

Parameter correction is a common practice in the gas turbine community, and the theoretical values of the exponents $a$ and $b$ for the steady-state and transient variables of interest can be found in many references (See, for instance, Refs. [21,22].) Yet, additional physical phenomena, such as the modification of the thermophysical properties of the working fluid, Reynolds number effects, and geometrical effects (e.g., clearance and blade untwist), make the engine behavior deviate from the assumptions of the Mach number similarity. Hence, a fine tuning of the $a$ and $b$ exponents for each parameter involved in the BCM should be carried out as explained in Ref. [23] for improved accuracy.

The following operations allow the adaptation of the original $\mathrm{BCM}$ to any ambient conditions.

1. Get current state derivatives from the on-board model.

2. Compute corrected state derivatives.

3. Build corrected TI from corrected state derivatives according to Eq. (8).

4. Call the BCM with corrected TI on input; get $\mathbf{b}_{\mathbf{k}}$ and $\mathbf{R}_{\mathbf{b}, \mathbf{k}}$ on output.

5. "Decorrect" $\mathbf{b}_{\mathbf{k}}$ to current ambient conditions.

As can be noted in item 5, the mean bias $\mathbf{b}_{\mathbf{k}}$ is decorrected, but not its associated covariance matrix. Hence, we implicitly assume that the uncertainty in the mean bias is constant for different ambient conditions. It means that the highest contribution to the measure- 


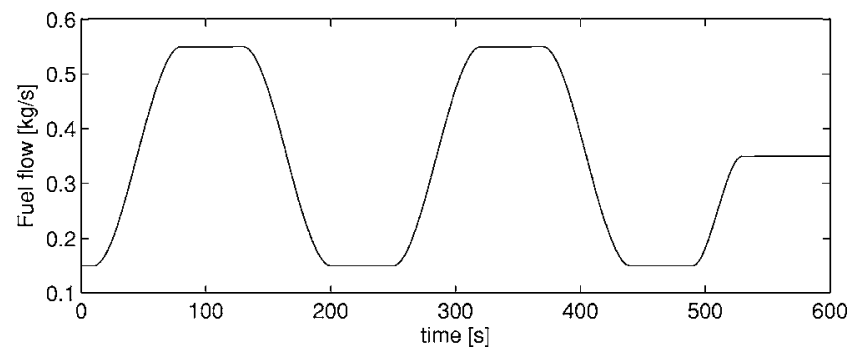

Fig. 11 Fuel flow profile, cruise conditions

ment covariance is the measurement noise (the magnitude of which is independent of the operating conditions) rather than the uncertainty in the mean bias. It is worth mentioning that these adaptations are applied outside of the core of the BCM, which is thereby not affected at all.

Test-Case B: Validation for Non-Sea-Level Static Conditions. To validate the correction procedure applied to the $\mathrm{BCM}$, a second test case has been designed. Cruise flight conditions (altitude $=10,800 \mathrm{~m}$, flight Mach number $=0.82$ ) are assumed. The simulated fault is the same engine deterioration as previously described. The open-loop scheduled fuel flow is plotted in Fig. 11 and is similar in shape to the one of test-case A. The reader will certainly notice the simplified nature of this test case, which is intended here for validation purposes only.

Figure 12 sketches the evolution of the identified health parameters when using the corrected BCM. It can be seen that the engine deterioration is accurately assessed (localization and magnitude). As for the identification under SLS conditions, one can notice slight oscillations in SW26R, SE26, and SW41R due to the remaining prediction error in $T_{3}$.

For the considered application, it can thus be stated that firstorder corrections brought by corrected parameters appear to be sufficient to use the original BCM, determined from SLS data, for monitoring the condition of the engine under other ambient conditions. It can be explained by the fact that the shortcomings of the model are intrinsic and hence do not depend on the atmospheric conditions. The proposed methodology for bias compensation is therefore very appealing, given that it is much easier to collect biases on the test bench than in flight.
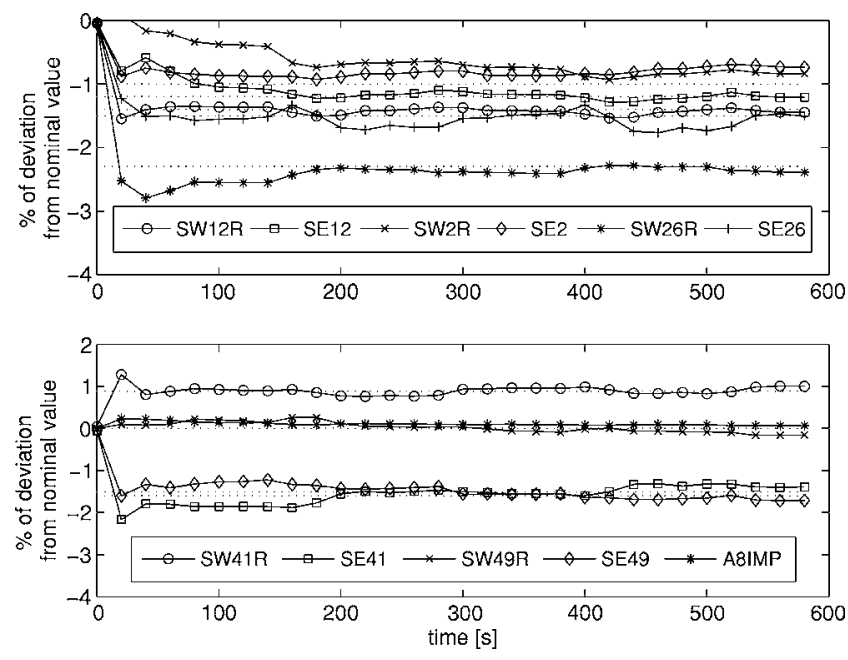

Fig. 12 Diagnosis with the corrected $\mathrm{BCM}$, cruise conditions

\section{Discussion}

The enhancement of the diagnosis capabilities possible with the $\mathrm{BCM}$ in the presence of model-plant mismatch has been discussed in the previous section. However, some more issues have to be discussed to complete the analysis of the results.

The first question is related to the continuity of the bias model with respect to the TI. In this paper, three different TI patterns have been defined, and both the mean bias $\mathbf{b}$ and the covariance $\mathbf{R}_{\mathbf{b}}$ are discontinuous between adjacent patterns. No instabilities of the DEKF have been noticed so far. It is supposed that the DEKF is unaware of those discontinuities because it is not sensitive to the derivative of the bias model. TI segments might be developed to match the specific acceleration and deceleration features of a particular control system (fuel control and actuators).

Another open question is linked to the complexity of the bias model. A very simple piecewise quadratic bias model has been considered in this application, and it has been shown to be sufficient for providing a rather accurate diagnosis. However, more complex models, such as neural networks, could be tested. Another research direction concerning the complexity of the bias model is the definition itself of that model. Throughout the paper, it has been assumed that the bias model only depends on the TI. A formulation with two input arguments such as TI and its time derivative, or TI and a state index, should be investigated. Data mining techniques could be used to this end.

An essential work is to further investigate the applicability of the BCM, defined from SLS mismatch data, throughout the flight envelope. This approach has been proven successful for the particular application considered in the present study, somewhat simplified with respect to real-world situations. Indeed, newgeneration engines are more complex, from the standpoint of architecture as well as control systems. The structure of the model-plant mismatch might then depend on the ambient and operating conditions, too. In that case, collection of mismatch data in an altitude test facility and/or on a flying test bed would become mandatory for a complete determination of the BCM.

Finally, some more studies still need to be undertaken concerning robustness issues. Those are twofold: First, the capability of the algorithm to cope with sensor malfunctions is still under development. Second, the applicability of the methodology presented herein has to be verified for other modeling errors such as sensor/actuator dynamics, fluid dynamics effects, bleed air and power take-offs, and especially biased steady-state engine modeling.

\section{Conclusion}

The ability to perform a reliable diagnosis in transient operation with an imperfect model of a gas turbine has been investigated. A methodology has been developed to compensate for the bias induced by model-plant mismatch by treating it as a pseudoGaussian variable. The improvements to the quality of the diagnosis with the new algorithm have been demonstrated on simple, but realistic test cases.

More specifically, it has been pointed out that taking into account both the mean bias and its related uncertainty improves the identification procedure in terms of stability and accuracy even with a rather simple structure of the bias model. The BCM, built from data gathered on a test bench, has also shown interesting generalization properties in order to carry out health monitoring for other ambient conditions. A simple approach relying on corrected parameters addresses this issue for the simulated test data available.

\section{Acknowledgment}

The authors wish to thank the reviewers and the Associate Editor for their valuable comments during the review process. 


\section{Nomenclature}

$\hat{\mathbf{a}}=$ estimation of an unknown variable $\mathbf{a}$

A8IMP $=$ nozzle exit area (nominal value: $1.4147 \mathrm{~m}^{2}$ )

$\mathbf{b}_{k}=$ mean of the model bias

EGT $=$ exhaust gas temperature

$k=$ discrete time index

$P_{i}=$ total pressure at station $i$

$\mathbf{R}_{\mathbf{b}, \mathbf{k}}=$ covariance matrix of the model bias

$\mathrm{SEi}=$ efficiency scaler of the component whose entry is located at section $i$ (nominal value: 1.0)

$\mathrm{SWiR}=$ flow capacity scaler of the component whose entry is located at section $i$ (nominal value: 1.0)

$T_{i}=$ total temperature at station $i$

$\mathbf{u}_{k}=$ actual command parameters

$\mathbf{v}_{k}=$ actual external disturbances

$\mathbf{w}_{k}=$ actual but unknown health parameters

$\mathbf{x}_{k}=$ actual but unknown state variables

$\mathbf{y}_{k}=$ observed measurements

$\boldsymbol{\epsilon}_{k}=$ measurement noise vector

$\boldsymbol{\nu}_{k}=$ process noise vector

$\mathcal{N}(\mathbf{m}, \mathbf{R})=$ a Gaussian probability density function with mean $\mathbf{m}$ and covariance matrix $\mathbf{R}$

\section{References}

[1] Volponi, A. J., 2003, Foundation of Gas Path Analysis (Part I and II), Gas Turbine Condition Monitoring and Fault Diagnosis, Lecture Series No. 2003 01, von Karman Institute for Fluid Dynamics, Brussels, Belgium.

[2] Kalman, R. E., and Bucy, R. S., 1961, "New Results in Linear Filtering and Prediction Theory," ASME J. Basic Eng., 83, pp. 95-107.

[3] Dewallef, P., 2005, "Application of the Kalman Filter to Health Monitoring of Gas Turbine Engines: A Sequential Approach to Robust Diagnosis," Ph.D. thesis, University of Liège.

[4] Urban, L. A., 1972, "Gas Path Analysis Applied to Turbine Engine Condition Monitoring," Eighth Joint Propulsion Specialist Conference, Paper No. 721082.

[5] Duponchel, J.-P., Loisy, J., and Carillo, R., 1992, "Steady and Transient Performance Calculation Method for Prediction, Analysis and Identification," Paper No. AGARD LS-183.
[6] Grönstedt, T., 2005, "Least Squares Based Transient Nonlinear Gas Path Analysis," ASME Paper No. GT2005-68717.

[7] Ogaji, S., Li, Y., Sampath, S., and Singh, R., 2003, "Gas Path Fault Diagnosis of a Turbofan Engine from Transient Data Using Artificial Neural Networks," ASME Paper No. GT2003-38423.

[8] Simon, D., and Simon, D. L., 2003, "Aircraft Turbofan Engine Health Estimation Using Constrained Kalman Filtering," ASME Paper No. GT2003-38584.

[9] Dewallef, P., and Léonard, O., 2003, "On-Line Performance Monitoring an Engine Diagnostic Using Robust Kalman Filtering Techniques," ASME Paper No. GT2003-38379.

[10] Borguet, S., Dewallef, P., and Léonard, O., 2005, "On-Line Transient Engine Diagnostics in a Kalman Filtering Framework," ASME Paper No. GT200568013.

[11] Volponi, A. J., 2005, "Use of Hybrid Engine Modeling for On-Board Module Performance Tracking," ASME Paper No. GT2005-68169.

[12] RTO, 2002, "Performance Prediction and Simulation of Gas Turbine Engine Operation," Research and Technology Organisation, Technical Report No. 44

[13] Nielsen, A. E., Moll, C. W., and Staudacher, S., 2005, "Modeling and Validation of the Thermal Effects on Gas Turbine Transients," ASME J. Eng. Gas Turbines Power, 127, pp. 564-572.

[14] Wan, E., and van der Merwe, R., 2001, "The Unscented Kalman Filter," Kalman Filtering and Neural Networks, Wiley Series on Adaptive and Learning Systems for Signal Processing, Communications and Control, Wiley, New York.

[15] Nelson, A. T., 2000, "Nonlinear Estimation and Modeling of Noisy Time Series by Dual Kalman Filtering Methods," Ph.D. thesis, Oregon Graduate Institute of Technology.

[16] Bishop, C. M., 1995, Neural Networks for Pattern Recognition, Clarendon, Oxford.

[17] Roth, B. A., Doel, D. L., and Cissell, J. J., 2005, "Probabilistic Matching of Turbofan Engine Performance Models to Test Data," ASME Paper No. GT2005-68201.

[18] Cerri, G., Borghetti, S., and Salvini, C., 2005, "Inverse Methodologies for Actual Status Recognition of Gas Turbine Components," ASME Paper No. PWR2005-50033.

[19] Stamatis, A., Mathioudakis, K., Ruiz, J., and Curnock, B., 2001, "Real-Time Engine Model Implementation for Adaptive Control and Performance Monitoring of Large Civil Turbofans," ASME Paper No. 2001-GT-0362.

[20] Curnock, B., 2000, “Obidicote Project -WP4: Steady-State Test Cases," RollsRoyce plc, Technical Report No. DNS62433.

[21] Walsh, P. P., and Fletcher, P., 1998, Gas Turbine Performance, Blackwell Science, London.

[22] Volponi, A. J., 1999, "Gas Turbine Parameter Corrections," ASME J. Eng. Gas Turbines Power, 121, pp. 613-621.

[23] Kurzke, J., 2003, "Model Based Gas Turbine Parameter Corrections," ASME Paper No. GT2003-38234. 